\title{
Poor Prognostic Biochemical Markers Predicting Fatalities Caused by COVID-19: A Retrospective Observational Study From a Developing Country
}

Muhammad Sohaib Asghar ${ }^{1}$, Syed J. Haider Kazmi ${ }^{2}$, Noman A. Khan ${ }^{3}$, Mohammed Akram ${ }^{4}$, Maira Hassan ${ }^{4}$, Uzma Rasheed ${ }^{4}$, Salman Ahmed Khan ${ }^{5}$

1. Internal Medicine, Dow International Medical College, Dow University Hospital, Dow University of Health Sciences, Karachi, PAK 2. Emergency Medicine, Liaquat National Hospital, Karachi, PAK 3. General Surgery, Liaquat National Hospital, Karachi, PAK 4. Internal Medicine, Liaquat National Hospital, Karachi, PAK 5. Internal Medicine, Dow International Medical College, Karachi, PAK

Corresponding author: Muhammad Sohaib Asghar, sohaib_asghar123@yahoo.com

\begin{abstract}
Background and objectives

Infections with severe acute respiratory syndrome coronavirus 2 (SARS-CoV-2) are rapidly spreading, posing a serious threat to the health of people worldwide, resulting in the World Health Organization officially declaring it a pandemic. There are several biochemical markers linked with predicting the severity of coronavirus disease. This study aims to identify the most effective predictive biomarker such as C-reactive protein (CRP), ferritin, lactate dehydrogenase (LDH), procalcitonin (PCT), and D-dimer, among others, in predicting the clinical outcome of the disease.
\end{abstract}

Materials and methods

This study was conducted as a retrospective, observational, multi-centric study, including all admitted COVID-19 positive patients only. The disease outcome was followed along with the hospital course of every patient at the time of analysis. Baseline laboratory investigations of all patients were monitored both at admission and discharge. A comparative analysis was done between the survivors $(n=263)$ and non-survivors $(n=101)$. Statistical analysis was conducted using IBM SPSS Statistics for Windows Version 25 (Armonk, NY: IBM Corp.).

Results

Of 364 patients, $65.7 \%$ were in the isolation ward, and $34.3 \%$ were in the intensive care unit; $72.3 \%$ of patients survived, while $27.7 \%$ of patients died. The mean age of the study population was $52.6 \pm 15.8$ years with female patients significantly younger than male patients $(\mathrm{p}=0.001)$ and 50 to 75 years being the most common age group ( $\mathrm{p}=0.121)$. Among the survivors versus non-survivors of COVID-19, there were significant differences in total leukocyte count $(\mathrm{p}<0.001)$, neutrophil count, $(\mathrm{p}<0.001)$, lymphocyte count $(p<0.001)$, urea $(p<0.001)$, serum bicarbonate $(p=0.001)$, CRP levels $(p<0.001), L D H ~(p=0.013)$, and D-dimer $(\mathrm{p}<0.001)$ at admission. At discharge, the laboratory values of non-surviving patients showed significant leukocytosis $(\mathrm{p}<0.001)$, neutrophilia $(\mathrm{p}<0.001)$, lymphocytopenia $(\mathrm{p}<0.001)$, decreased monocytes $(\mathrm{p}<0.001)$, elevated urea and creatinine $(\mathrm{p}<0.001)$, hypernatremia $(\mathrm{p}<0.001)$, decreased serum bicarbonate levels $(\mathrm{p}<0.001)$, elevated CRP level $(\mathrm{p}=0.040), \mathrm{LDH}(\mathrm{p}<0.001)$, ferritin $(\mathrm{p}=0.001)$, and $\mathrm{D}-\operatorname{dimer}(\mathrm{p}<0.001)$.

Among the recovered patients, the laboratory investigations at admission were significantly different from those at discharge like increased platelets $(\mathrm{p}=0.007)$, lower neutrophil count $(\mathrm{p}=0.001)$, higher lymphocyte count $(\mathrm{p}=0.005)$, an improved creatinine $(\mathrm{p}=0.020)$, higher sodium $(\mathrm{p}=0.008)$, increased bicarbonate levels $(\mathrm{p}<0.001)$, decreased CRP levels $(\mathrm{p}<0.001)$, and a lower LDH $(\mathrm{p}=0.039)$. However, the laboratory values of non-surviving patients had shown a lower hemoglobin $(\mathrm{p}=0.016)$, increased mean cell volume $(\mathrm{p}<0.001)$, significantly increased total leukocyte count $(\mathrm{p}<0.001)$, increased urea and creatinine $(\mathrm{p}<0.001)$, hypernatremia $(\mathrm{p}<0.001)$, increased bicarbonate $(\mathrm{p}=0.025)$, elevated $\mathrm{D}$-dimer levels $(\mathrm{p}=0.043)$, and elevated PCT $(p=0.021)$ on discharge. Receiver operating characteristic analysis concluded LDH (area under the curve [AUC]: 0.875), D-dimer (AUC: 0.803), and PCT (AUC: 0.769) were superior biomarkers to ferritin (AUC: 0.714) and CRP (AUC: 0.711) in predicting the fatality of COVID-19.

Conclusion

Inflammatory markers are a useful guide for predicting mortality, and the study results concluded that LDH, PCT, D-dimer, CRP, and ferritin were effective biomarkers.

Categories: Internal Medicine, Allergy/Immunology, Infectious Disease 
Keywords: covid-19, coronavirus, biomarkers, pandemic, mortality, infectious diseases, pakistan, severity, prognosis, survivor

\section{Introduction}

In December 2019, a mysterious pneumonia-like syndrome was observed in Wuhan, China [1]. The causative virus was officially termed as the severe acute respiratory syndrome coronavirus 2 (SARS-CoV-2), and the disease it caused was named coronavirus disease (COVID-19) by The International Committee on Taxonomy of Viruses [2]. COVID-19 is primarily a respiratory disease but can cause various non-respiratory manifestations, such as gastrointestinal, neurological, renal, and cardiovascular symptoms [3]. The SARSCoV-2 infection is rapidly spreading, posing a serious threat to the health of people worldwide, resulting in the World Health Organization officially declaring it a pandemic on March 11, 2020.

There are several biochemical markers linked with predicting the severity of coronavirus disease. Ferritin, produced in inflammatory conditions of the body (infectious, malignant, hematologic, and rheumatologic), is an important acute phase reactant. Microscopically, ferritin plays a defensive role within the body by limiting the supply of iron, due to which elevated serum ferritin levels can be seen in individuals with high pathogenic loads [4]. Ferritin not only limits the availability of iron to the pathogen but also regulates cytokine synthesis and release that are responsible for the cytokine (proinflammatory) storm [5].

C-reactive protein (CRP) is an acute-phase reactant that is synthesized by the liver in response to inflammation or infection. Unlike most acute-phase proteins that undergo large variations in plasma levels (depending on the synthesis, consumption, and catabolism rates), plasma CRP levels remain nearly constant. During acute inflammation, serum concentrations increase dramatically, making it a more accurate marker for sepsis [6]. CRP also contributes to the proinflammatory cycle by activating inflammatory cytokines in the body [7].

CRP and serum ferritin both play important roles in producing proinflammatory cytokines. Interestingly, the principal finding of immunopathology in COVID-19 is the cytokine storm. The virus replicates rapidly in the body's endothelial and epithelial cells, resulting in the immune system developing significant numbers of proinflammatory cytokines and chemokines [1]. The severity of COVID-19 resides in the development of large quantities of proinflammatory cytokines that can eventually contribute to acute respiratory distress syndrome (ARDS) and multiple organ failure (MOF) [8]. Studies have shown that respiratory tract viral infections were associated with poor clinical outcomes due to the high rates of cytokines and chemokines released during the infection [9]. Severe COVID-19 cases are progressing quickly to complications, such as ARDS, sepsis, septic shock, metabolic acidosis, coagulopathy, and MOF [10,11]. A study that analyzed the clinical characteristics of deceased coronavirus patients identified sepsis, ARDS, respiratory failure, and heart failure as the most critical complications [12].

Due to its valuable role in the diagnosis and prognosis regarding sepsis, procalcitonin (PCT) is widely considered to be the most useful marker of severe systemic inflammation. Under normal circumstances, PCT is produced in the C-cells of the parathyroid gland. However, the rise in PCT seen during infectious states is believed to stem from neuroendocrine cells in the lungs and intestine. Its release is mediated by proinflammatory cytokines, such as tumor necrosis factor-alpha and interleukin-6. [13]. Studies found that PCT levels were lower in seriously ill patients with viral infections and were much higher in bacterial infections [14]. COVID-19 patients with elevated levels of PCT were associated with a five-fold greater risk of severe disease progression [15]. It may be because secondary bacterial infections are also common in COVID-19 pneumonia.

Severe sepsis patients also have associated high lactate dehydrogenase (LDH) levels. LDH is a cellular injury marker that shows the extent of damage to the tissue. Failure of LDH levels to normalize within 48 hours of the onset of sepsis is a strong predictor of patient mortality [16].

During sepsis, there is an upregulation of tissue factor resulting in a downregulation of anti-thrombin and a subsequent increase in plasma thrombin. At the same time, the production of protein $\mathrm{C}$ decreases, and upregulation of type 1 plasminogen activator inhibitor further inhibits fibrinolysis. Collectively, all these changes induce a hypercoagulable state. Increased coagulation and hypotension in sepsis can result in MOF, which is the most severe and life-threatening consequence of sepsis. A variety of molecules play important roles in the activation of the coagulation cascade. D-dimer is a sign of ongoing active fibrinolysis and, therefore, also of coagulation [17]. D-dimer is a measure of the coagulation cascade and assesses the severity of the host response, which led it to play an important role in the risk stratification of patients with sepsis to improve clinical management. A study showed that the higher the D-dimer levels, the greater the risk of sepsis and septic shock for the patient [18].

This study aims to identify the roles of these biomarkers (CRP, ferritin, LDH, PCT, and D-dimer), among others, in predicting the severity and clinical outcome during the disease. The identification of an effective and predictive biomarker would thus help in risk stratifying the patients and overall improving the clinical management of patients with COVID-19, especially in the region. 


\section{Cureus}

\section{Materials And Methods}

This study was conducted as a retrospective, observational, multi-centric study, including all the admitted COVID-19 positive patients only. The outcome of the disease was followed along with the hospital course of every patient at the time of analysis. Baseline laboratory investigations of all patients were monitored both at admission and discharge. The comparative analysis was done between the survivors $(n=263)$ and nonsurvivors ( $\mathrm{n}=101)$. The statistical analysis was conducted using IBM SPSS Statistics for Windows, Version 25 (Armonk, NY: IBM Corp.). All continuous variables were described as mean and standard deviation, and then compared using independent sample t-test and Mann-Whitney U test accordingly. Receiver operating characteristic (ROC) curves were used to determine the predictability of biochemical markers for the outcome of the disease. The Youden index was used as a summary measure of cut-off values for area under the curve (AUC). A p-value of $<0.05$ was considered statistically significant. All the highly significant values of $<0.001$ were rounded off as 0.001 .

\section{Results}

A total of 364 COVID-19 positive patients were included in the study with a mean age of $52.6 \pm 15.8$ years with female patients (mean age: $48.4 \pm 16.4$ ) significantly younger than male patients (mean age: $54.6 \pm 15.2$; $\mathrm{p}=0.001$ ). The most common age group was 50-75 years, with two-thirds of patients being males $(\mathrm{p}=0.121)$. The majority of the patients were experiencing mild to moderate symptoms and were therefore admitted to the isolation ward (65.7\%), while the remaining $34.3 \%$ were experiencing more severe disease and were admitted to the intensive care unit. The descriptive statistics of the study population are stated in Table 1. 


\section{Cureus}

\begin{tabular}{|c|c|c|c|c|c|}
\hline No. & Characteristics & Total $(n=364)$ & Survivors (n=263) & Non-Survivors ( $n=101$ ) & P-value \\
\hline \multirow{2}{*}{1} & Median age (IQR) & $55.00(43.00-65.00)$ & $50.00(39.25-60.75)$ & $63.00(55.00-70.00)$ & $<0.001^{*}$ \\
\hline & Mean age (in years) & $52.69 \pm 15.88$ & $49.33 \pm 16.14$ & $61.02 \pm 11.63$ & $<0.001^{\star \star}$ \\
\hline \multirow{2}{*}{2} & Males ( $n=246)$, Median (IQR) & $56.00(45.00-67.00)$ & $51.00(43.00-64.00)$ & $63.00(57.00-70.00)$ & $<0.001^{*}$ \\
\hline & Mean \pm SD & $54.63 \pm 15.28$ & $51.63 \pm 15.78$ & $62.50 \pm 10.43$ & $<0.001^{\star \star}$ \\
\hline \multirow{2}{*}{3} & Females ( $n=118)$, Median (IQR) & $52.00(38.00-60.00)$ & $43.00(30.25-55.75)$ & $60.00(50.50-68.50)$ & $<0.001^{*}$ \\
\hline & Mean \pm SD & $48.41 \pm 16.41$ & $43.95 \pm 15.80$ & $58.15 \pm 13.38$ & $<0.001^{\star \star}$ \\
\hline \multirow{5}{*}{4} & Age groups & & & & - \\
\hline & $0-25$ & $16(4.5 \%)$ & $14(5.3 \%)$ & $2(2.0 \%)$ & \multirow{4}{*}{$<0.001^{\dagger}$} \\
\hline & $26-50$ & $135(37.1 \%)$ & $119(45.2 \%)$ & $16(15.8 \%)$ & \\
\hline & $51-75$ & $198(54.3 \%)$ & $120(45.6 \%)$ & $78(77.2 \%)$ & \\
\hline & $>75$ & $15(4.2 \%)$ & $10(3.8 \%)$ & $5(4.9 \%)$ & \\
\hline \multirow{3}{*}{5} & Hospital stay & & & & - \\
\hline & Isolation ward & $239(65.7 \%)$ & $212(80.6 \%)$ & $27(26.7 \%)$ & \multirow{2}{*}{$<0.001^{\dagger}$} \\
\hline & ICU & $125(34.3 \%)$ & $51(19.4 \%)$ & $74(73.3 \%)$ & \\
\hline \multirow{4}{*}{6} & Recovered patients $(n=263)$ & & \multicolumn{2}{|c|}{ Expired patients $(\mathrm{n}=101)$} & - \\
\hline & Males: 180 (73.2\%) & Females: 83 (70.3\%) & Males: 66 (26.8\%) & Females: 35 (29.7\%) & $0.572^{\dagger}$ \\
\hline & Ward: 144 (87.8\%) & Ward: 68 (90.7\%) & Ward: 20 (12.2\%) & Ward: 7 (9.3\%) & $0.517^{\dagger}$ \\
\hline & ICU: 36 (43.9\%) & ICU: 15 (34.9\%) & ICU: 46 (56.1\%) & ICU: 28 (65.1\%) & $0.330^{\dagger}$ \\
\hline
\end{tabular}

\section{TABLE 1: Demographic data of the study population $(n=364)$}

*Mann-Whitney $U$ test to compute the p-value.

**Indicates independent sample t-test used to compute the p-value.

†Chi-square test used to compute the p-value.

IQR, interquartile range; ICU, intensive care unit; SD, standard deviation.

Among the survivors versus non-survivors of COVID-19, there were significant differences in the baseline laboratory investigations at admission, including mean hemoglobin $(\mathrm{p}=0.066)$, total leukocyte count (TLC) $(\mathrm{p}<0.001)$, neutrophil count, $(\mathrm{p}<0.001)$, lymphocyte count $(\mathrm{p}<0.001)$, monocyte count $(\mathrm{p}=0.073)$, urea $(\mathrm{p}<0.001)$, creatinine $(\mathrm{p}=0.030)$, serum bicarbonate $(\mathrm{p}=0.001)$, CRP levels $(\mathrm{p}<0.001), \mathrm{LDH}(\mathrm{p}=0.013)$, ferritin $(\mathrm{p}=0.066)$, D-dimer $(\mathrm{p}<0.001)$, and PCT $(\mathrm{p}=0.056)$. At discharge, the laboratory values of non-surviving patients showed thrombocytopenia $(\mathrm{p}=0.049)$, significant leukocytosis $(\mathrm{p}<0.001)$, neutrophilia $(\mathrm{p}<0.001)$, lymphocytopenia $(p<0.001)$, decreased monocytes $(p<0.001)$, elevated urea and creatinine $(p<0.001)$, hypernatremia $(\mathrm{p}<0.001)$, increased chloride $(\mathrm{p}=0.044)$, decreased serum bicarbonate levels $(\mathrm{p}<0.001)$, elevated CRP level $(\mathrm{p}=0.040)$, LDH $(\mathrm{p}<0.001)$, ferritin $(\mathrm{p}=0.001)$, D-dimer $(\mathrm{p}<0.001)$, and PCT $(\mathrm{p}=0.113)$ (Table 2). 


\begin{tabular}{|c|c|c|c|c|c|c|c|}
\hline \multirow{2}{*}{ No. } & \multirow{2}{*}{$\begin{array}{l}\text { Laboratory } \\
\text { investigation }\end{array}$} & Survivors & Non-survivors & \multirow{2}{*}{ P-value } & Survivors & Non-survivors & \multirow{2}{*}{ P-value } \\
\hline & & \multicolumn{2}{|l|}{ At admission } & & \multicolumn{2}{|l|}{ At discharge } & \\
\hline 1 & Hemoglobin & $12.18 \pm 2.40$ & $11.66 \pm 2.45$ & 0.066 & $11.54 \pm 2.30$ & $11.20 \pm 2.23$ & 0.279 \\
\hline 2 & MCV & $83.84 \pm 8.08$ & $81.57 \pm 9.46$ & 0.143 & $83.39 \pm 13.38$ & $84.49 \pm 11.38$ & 0.716 \\
\hline 3 & Platelets & $232.53 \pm 113.19$ & $233.73 \pm 103.85$ & 0.928 & $270.94 \pm 131.15$ & $232.17 \pm 147.94$ & $0.049^{\star}$ \\
\hline 4 & TLC & $9.50 \pm 5.19$ & $14.10 \pm 7.70$ & $<0.001^{*}$ & $10.13 \pm 4.48$ & $17.74 \pm 8.76$ & $<0.001^{*}$ \\
\hline 5 & Neutrophil & $70.77 \pm 13.06$ & $79.88 \pm 12.13$ & $<0.001^{*}$ & $71.26 \pm 13.07$ & $81.77 \pm 10.50$ & $<0.001^{*}$ \\
\hline 6 & Lymphocyte & $22.34 \pm 11.48$ & $14.26 \pm 9.49$ & $<0.001^{\star}$ & $21.44 \pm 12.13$ & $12.40 \pm 8.21$ & $<0.001^{*}$ \\
\hline 7 & Monocyte & $5.58 \pm 2.66$ & $4.85 \pm 3.73$ & 0.073 & $5.72 \pm 2.77$ & $4.37 \pm 1.99$ & $<0.001^{\star}$ \\
\hline 8 & Urea & $42.64 \pm 44.61$ & $70.15 \pm 51.83$ & $<0.001^{*}$ & $51.88 \pm 49.82$ & $142.45 \pm 74.94$ & $<0.001^{*}$ \\
\hline 9 & Creatinine & $1.50 \pm 2.55$ & $2.20 \pm 2.75$ & $0.030^{*}$ & $1.45 \pm 1.67$ & $3.38 \pm 2.49$ & $<0.001^{*}$ \\
\hline 10 & Sodium & $138.29 \pm 5.51$ & $138.77 \pm 7.45$ & 0.511 & $139.12 \pm 4.92$ & $147.72 \pm 9.48$ & $<0.001^{\star}$ \\
\hline 11 & Potassium & $4.09 \pm 0.75$ & $4.08 \pm 0.81$ & 0.890 & $4.73 \pm 9.58$ & $5.70 \pm 11.26$ & 0.534 \\
\hline 12 & Chloride & $103.81 \pm 5.92$ & $102.47 \pm 7.29$ & 0.074 & $101.08 \pm 10.82$ & $105.24 \pm 14.79$ & $0.044^{*}$ \\
\hline 13 & Bicarbonate & $20.61 \pm 3.40$ & $18.98 \pm 4.35$ & $0.001^{*}$ & $22.66 \pm 3.93$ & $20.18 \pm 5.03$ & $0.001^{*}$ \\
\hline 14 & CRP & $113.28 \pm 108.74$ & $198.67 \pm 121.54$ & $<0.001^{*}$ & $56.84 \pm 84.79$ & $182.94 \pm 507.36$ & $0.040^{\star}$ \\
\hline 15 & LDH & $495.62 \pm 279.68$ & $881.12 \pm 1398.56$ & $0.013^{\star}$ & $465.69 \pm 179.32$ & $\begin{array}{l}1298.92 \pm \\
1810.82\end{array}$ & $<0.001^{*}$ \\
\hline 16 & Ferritin & $1463.36 \pm 4840.10$ & $\begin{array}{l}2757.42 \pm \\
6483.39\end{array}$ & 0.066 & $\begin{array}{l}1130.40 \pm \\
1501.52\end{array}$ & $\begin{array}{l}3462.06 \pm \\
4280.59\end{array}$ & $0.001^{*}$ \\
\hline 17 & D-dimer & $4.09 \pm 8.07$ & $11.58 \pm 15.83$ & $<0.001^{*}$ & $3.94 \pm 6.88$ & $14.56 \pm 17.11$ & $<0.001^{*}$ \\
\hline 18 & Procalcitonin & $1.45 \pm 8.48$ & $4.66 \pm 12.09$ & 0.056 & $3.62 \pm 14.80$ & $14.83 \pm 28.69$ & 0.113 \\
\hline 19 & Troponin I & $19.54 \pm 69.82$ & $35.65 \pm 104.14$ & 0.391 & $40.02 \pm 74.49$ & $57.95 \pm 125.81$ & 0.722 \\
\hline 20 & Pro-BNP & $\begin{array}{l}11779.76 \pm \\
44060.92\end{array}$ & $\begin{array}{l}4374.70 \pm \\
6862.73\end{array}$ & 0.324 & $\begin{array}{l}4427.70 \pm \\
6746.50\end{array}$ & $\begin{array}{l}4769.50 \pm \\
5326.63\end{array}$ & 0.956 \\
\hline 21 & Albumin & $2.93 \pm 0.73$ & $3.09 \pm 0.62$ & 0.486 & - & - & - \\
\hline 22 & ESR & $46.50 \pm 28.80$ & $63.16 \pm 50.94$ & 0.174 & - & - & - \\
\hline 23 & Fibrinogen & $446.66 \pm 190.61$ & $500.56 \pm 248.21$ & 0.579 & - & - & - \\
\hline
\end{tabular}

TABLE 2: Comparison of baseline laboratory investigations (means) among patients with COVID$19(n=364)$

All p-values calculated by independent sample t-test (*significant values of $<0.05$ ).

COVID-19, coronavirus disease 2019; MCV, mean corpuscular volume; TLC, total leukocyte count; CRP, C-reactive protein; LDH, lactate dehydrogenase; BNP, B-type natriuretic peptide; ESR, erythrocyte sedimentation rate.

Among the recovered patients, the laboratory investigations at admission were significantly different from those at discharge like decreased hemoglobin $(\mathrm{p}=0.020)$, increased platelets $(\mathrm{p}=0.007)$, lower neutrophil count ( $\mathrm{p}=0.001)$, higher lymphocyte count $(\mathrm{p}=0.005)$, mildly increased monocyte count $(\mathrm{p}=0.049)$, an improved creatinine $(\mathrm{p}=0.020)$, higher sodium $(\mathrm{p}=0.008)$, lower chloride $(\mathrm{p}=0.038)$, increased bicarbonate levels ( $p<0.001)$, decreased CRP levels $(\mathrm{p}<0.001)$, and a lower LDH $(\mathrm{p}=0.039)$. The laboratory values of nonsurviving patients had shown a lower hemoglobin $(\mathrm{p}=0.016)$, increased mean cell volume $(\mathrm{p}<0.001)$, significantly increased TLC $(\mathrm{p}<0.001)$, neutrophilia $(\mathrm{p}=0.074)$, thrombocytopenia $(\mathrm{p}=0.048)$, increased urea and creatinine $(\mathrm{p}<0.001)$, hypernatremia $(\mathrm{p}<0.001)$, increased bicarbonate $(\mathrm{p}=0.025)$, elevated $\mathrm{D}$-dimer levels $(\mathrm{p}=0.043)$, and elevated PCT $(\mathrm{p}=0.021)$ on discharge (Table 3). 


\begin{tabular}{|c|c|c|c|c|c|c|c|}
\hline \multirow{2}{*}{ No. } & \multirow{2}{*}{ Laboratory investigation } & \multicolumn{2}{|l|}{ Survivors ( $(\mathbf{n}=263$ ) } & \multirow{2}{*}{ P-value } & \multicolumn{2}{|c|}{ Non-Survivors ( $n=101)$} & \multirow{2}{*}{ P-value } \\
\hline & & At admission & At discharge & & At admission & At expiry & \\
\hline 1 & Hemoglobin & $11.93 \pm 2.29$ & $11.60 \pm 2.30$ & $0.020^{*}$ & $11.75 \pm 2.41$ & $11.22 \pm 2.22$ & $0.016^{\star}$ \\
\hline 2 & MCV & $83.46 \pm 9.85$ & $83.33 \pm 13.49$ & 0.931 & $80.30 \pm 10.26$ & $84.49 \pm 11.38$ & $<0.001^{*}$ \\
\hline 3 & Platelets & $239.66 \pm 124.79$ & $270.37 \pm 129.57$ & $0.007^{*}$ & $239.04 \pm 99.54$ & $233.85 \pm 145.48$ & 0.717 \\
\hline 4 & TLC & $10.52 \pm 6.05$ & $10.12 \pm 4.46$ & 0.417 & $12.80 \pm 6.09$ & $17.83 \pm 8.69$ & $<0.001^{*}$ \\
\hline 5 & Neutrophil & $74.63 \pm 11.66$ & $70.90 \pm 13.40$ & $0.001^{*}$ & $79.79 \pm 10.94$ & $81.87 \pm 10.35$ & 0.074 \\
\hline 6 & Lymphocyte & $19.16 \pm 10.62$ & $21.79 \pm 12.38$ & $0.005^{*}$ & $14.25 \pm 7.70$ & $12.34 \pm 8.13$ & $0.048^{\star}$ \\
\hline 7 & Monocyte & $5.15 \pm 2.54$ & $5.73 \pm 2.76$ & $0.049^{*}$ & $4.90 \pm 3.74$ & $4.34 \pm 1.97$ & 0.184 \\
\hline 8 & Urea & $52.02 \pm 51.93$ & $51.08 \pm 48.95$ & 0.781 & $71.64 \pm 54.08$ & $140.02 \pm 74.57$ & $<0.001^{*}$ \\
\hline 9 & Creatinine & $1.83 \pm 3.09$ & $1.42 \pm 1.64$ & $0.020^{*}$ & $2.21 \pm 2.84$ & $3.33 \pm 2.46$ & $<0.001^{*}$ \\
\hline 10 & Sodium & $137.64 \pm 6.80$ & $139.15 \pm 4.82$ & $0.008^{\star}$ & $138.05 \pm 8.21$ & $147.78 \pm 9.29$ & $<0.001^{\star}$ \\
\hline 11 & Potassium & $4.22 \pm 0.94$ & $4.69 \pm 9.37$ & 0.594 & $4.10 \pm 0.84$ & $5.64 \pm 11.03$ & 0.236 \\
\hline 12 & Chloride & $103.24 \pm 7.15$ & $101.09 \pm 10.61$ & $0.038^{\star}$ & $103.61 \pm 11.73$ & $105.14 \pm 14.51$ & 0.446 \\
\hline 13 & Bicarbonate & $20.03 \pm 3.66$ & $22.70 \pm 4.17$ & $<0.001^{x}$ & $18.80 \pm 3.99$ & $20.25 \pm 4.98$ & $0.025^{\star}$ \\
\hline 14 & CRP & $130.19 \pm 105.48$ & $56.84 \pm 84.79$ & $<0.001^{*}$ & $201.75 \pm 121.21$ & $182.94 \pm 507.36$ & 0.751 \\
\hline 15 & LDH & $499.20 \pm 188.40$ & $456.69 \pm 179.32$ & $0.039^{*}$ & $841.47 \pm 1618.16$ & $\begin{array}{l}1298.92 \pm \\
1810.82\end{array}$ & 0.137 \\
\hline 16 & Ferritin & $1409.19 \pm 2012.51$ & $\begin{array}{l}1130.40 \pm \\
1501.52\end{array}$ & 0.160 & $\begin{array}{l}2192.10 \pm \\
6300.94\end{array}$ & $\begin{array}{l}3462.06 \pm \\
4820.59\end{array}$ & 0.169 \\
\hline 17 & D-dimer & $4.68 \pm 9.47$ & $3.94 \pm 6.88$ & 0.456 & $9.08 \pm 13.54$ & $14.56 \pm 17.11$ & $0.043^{\star}$ \\
\hline 18 & Procalcitonin & $4.44 \pm 17.30$ & $3.62 \pm 14.80$ & 0.178 & $3.41 \pm 11.32$ & $14.83 \pm 28.69$ & $0.021^{*}$ \\
\hline 19 & Troponin I & $48.48 \pm 131.09$ & $40.02 \pm 74.49$ & 0.780 & $80.17 \pm 173.76$ & $57.95 \pm 125.81$ & 0.420 \\
\hline 20 & Pro-BNP & $\begin{array}{l}8457.10 \pm \\
10539.14\end{array}$ & $\begin{array}{l}6449.05 \pm \\
8155.69\end{array}$ & 0.445 & $\begin{array}{l}4234.50 \pm \\
5055.10\end{array}$ & $\begin{array}{l}4769.50 \pm \\
5326.63\end{array}$ & 0.219 \\
\hline 21 & Fibrinogen & - & - & - & 421.00 & $476.50 \pm$ & 0.409 \\
\hline
\end{tabular}

TABLE 3: Progress of laboratory investigations during hospital stay of patients with COVID-19 $(n=364)$

P-value calculated by paired sample t-test (*significant values of $<0.05$ ).

COVID-19, coronavirus disease 2019; MCV, mean corpuscular volume; TLC, total leukocyte count; CRP, C-reactive protein; LDH, lactate dehydrogenase; BNP, B-type natriuretic peptide.

ROC analysis of admitting laboratory investigations for fatalities due to COVID-19 showed PCT at a cut-off value $0.12 \mathrm{ng} / \mathrm{ml}$ (AUC: $0.769, \mathrm{p}<0.001$ ), predicting death with a sensitivity of $85.5 \%$ and positive predictive value (PPV) of $83.3 \%$. D-dimer was shown at a cut-off value of $1.71 \mathrm{mcg} / \mathrm{ml}$ (AUC: $0.828, \mathrm{p}<0.001$ ), predicting death at $79.5 \%$ sensitivity and $82.5 \%$ PPV. Neutrophil counts were at a cut-off value of $72.50 \%$, predicting death at $87.5 \%$ sensitivity and $91.3 \%$ PPV (AUC: $0.733, \mathrm{p}<0.001$ ). LDH at a $629.50 \mathrm{U} / \mathrm{L}$ cut-off value (AUC: 0.723 , $\mathrm{p}<0.001$ ) predicted death with $59.8 \%$ sensitivity and $80.0 \%$ PPV, while urea (AUC: $0.726, \mathrm{p}<0.001$ ) at a cut-off value of $33.27 \mathrm{mg} / \mathrm{dl}$ and creatinine (AUC: $0.719, \mathrm{p}<0.001$ ) at a cut-off of $1.11 \mathrm{mg} / \mathrm{dl}$ have been shown predicting death at admission. However, CRP at a cut-off of $108.30 \mathrm{mg} / \mathrm{dl}$ (AUC: $0.701, \mathrm{p}<0.001$ ) and ferritin at a cut-off of $1658.0 \mathrm{ng} / \mathrm{ml}$ (AUC: $0.636, \mathrm{p}<0.001$ ) has not been showing death predictability with good sensitivity as compared to the above parameters. ROC curves for non-surviving patients at discharge showed $\mathrm{LDH}$ at a cut-off value of $611.0 \mathrm{U} / \mathrm{L}$ (AUC: $0.875, \mathrm{p}<0.001$ ) is predicting death at $81.0 \%$ sensitivity and $86.7 \%$ PPV. Urea at a cut-off of $60.92 \mathrm{mg} / \mathrm{dl}$ (AUC: $0.860, \mathrm{p}<0.001$ ) has shown predicting death with a sensitivity of $83.6 \%$ and PPV of $88.7 \%$. Creatinine at a cut-off of $1.35 \mathrm{mg} / \mathrm{dl}$ (AUC: $0.827, \mathrm{p}<0.001$ ) predicted death at $78.1 \%$ 
sensitivity and $85.8 \%$ PPV. D-dimer at a cut-off of $1.89 \mathrm{mcg} / \mathrm{ml}$ (AUC: $0.803, \mathrm{p}=0.848$ ) predicted death at 86.2\% and PPV 86.7\%. Sodium at a cut-off of $145.50 \mathrm{mEq} / \mathrm{L}$ (AUC: 0.791 , p $<0.001$ ), TLC at a cut-off of $12.90 \mathrm{x}$ $10^{9} / \mathrm{L}$, neutrophil at a cut-off of $76.50 \%$ (AUC: $0.765, \mathrm{p}<0.001$ ), and PCT at a cut-off of $0.51 \mathrm{ng} / \mathrm{dl}$ (AUC: $0.755, \mathrm{p}=0.002$ ) have been shown predicting fatality, while ferritin at a cut-off of $909 \mathrm{ng} / \mathrm{ml}$ (AUC: 0.714 , $\mathrm{p}<0.001$ ) and CRP at a cut-off of $35.60 \mathrm{mg} / \mathrm{dl}$ (AUC: $0.711, \mathrm{p}<0.001$ ) again fall behind the above markers in predicting death at discharge (Table 4; Figures 1,2).

\begin{tabular}{|c|c|c|c|c|c|c|c|c|c|}
\hline No. & Variable state & AUC & SE & $95 \% \mathrm{Cl}$ & Sensitivity & Specificity & PPV & NPV & P-value \\
\hline \multirow{3}{*}{1} & D-dimer (mcg/ml) & & & & & & & & \\
\hline & Admission (cut-off: 1.71) & 0.733 & 0.034 & $0.666-0.800$ & $79.5 \%$ & $59.3 \%$ & $82.5 \%$ & $54.5 \%$ & $<0.001$ \\
\hline & Discharge (cut-off: 1.89) & 0.803 & 0.037 & $0.730-0.875$ & $86.2 \%$ & $62.7 \%$ & $86.7 \%$ & $61.7 \%$ & $<0.001$ \\
\hline \multirow{3}{*}{2} & CRP (mg/dl) & & & & & & & & \\
\hline & Admission (cut-off: 108.30) & 0.701 & 0.032 & $0.639-0.763$ & $75.5 \%$ & $56.7 \%$ & $82.7 \%$ & $45.7 \%$ & $<0.001$ \\
\hline & Discharge (cut-off: 35.60) & 0.711 & 0.038 & $0.637-0.785$ & $66.7 \%$ & $68.1 \%$ & $77.1 \%$ & $55.8 \%$ & $<0.001$ \\
\hline \multirow{3}{*}{3} & LDH (U/L) & & & & & & & & \\
\hline & Admission (cut-off: 629.50) & 0.723 & 0.035 & $0.655-0.791$ & $59.8 \%$ & $79.1 \%$ & $80.0 \%$ & $58.4 \%$ & $<0.001$ \\
\hline & Discharge (cut-off: 611.00) & 0.875 & 0.030 & $0.817-0.934$ & $81.0 \%$ & $84.8 \%$ & $86.7 \%$ & $78.5 \%$ & $<0.001$ \\
\hline \multirow{3}{*}{4} & Ferritin (ng/ml) & & & & & & & & \\
\hline & Admission (cut-off: 1658.00) & 0.636 & 0.036 & $0.565-0.706$ & $41.6 \%$ & $81.5 \%$ & $74.3 \%$ & $52.1 \%$ & $<0.001$ \\
\hline & Discharge (cut-off: 909.00) & 0.714 & 0.045 & $0.625-0.803$ & $74.6 \%$ & $62.2 \%$ & $77.3 \%$ & $58.7 \%$ & $<0.001$ \\
\hline \multirow{3}{*}{5} & Procalcitonin (ng/ml) & & & & & & & & \\
\hline & Admission (cut-off: 0.12) & 0.769 & 0.038 & $0.695-0.842$ & $85.5 \%$ & $58.8 \%$ & $83.3 \%$ & $62.8 \%$ & $<0.001$ \\
\hline & Discharge (cut-off: 0.51 ) & 0.755 & 0.069 & $0.621-0.890$ & $62.5 \%$ & $80.0 \%$ & $57.1 \%$ & $83.3 \%$ & 0.002 \\
\hline \multirow{3}{*}{6} & Troponin I (pg/ml) & & & & & & & & \\
\hline & Admission (cut-off: 0.13 ) & 0.692 & 0.058 & 0.579-0.806 & $71.1 \%$ & $71.4 \%$ & $76.1 \%$ & $65.9 \%$ & 0.002 \\
\hline & Discharge (cut-off: 0.31) & 0.708 & 0.139 & $0.436-0.980$ & $91.7 \%$ & $62.5 \%$ & $83.3 \%$ & $78.6 \%$ & 0.123 \\
\hline \multirow{3}{*}{7} & Total leukocyte count $\left(\times 10^{9} / \mathrm{L}\right)$ & & & & & & & & \\
\hline & Admission (cut-off: 9.31) & 0.709 & 0.029 & $0.652-0.767$ & $72.1 \%$ & $58.9 \%$ & $84.2 \%$ & $41.0 \%$ & $<0.001$ \\
\hline & Discharge (cut-off: 12.90) & 0.786 & 0.035 & $0.718-0.853$ & $75.6 \%$ & $76.7 \%$ & $84.2 \%$ & $65.7 \%$ & $<0.001$ \\
\hline \multirow{3}{*}{8} & Neutrophil count (\%) & & & & & & & & \\
\hline & Admission (cut-off: 72.50) & 0.733 & 0.028 & $0.678-0.788$ & $87.5 \%$ & $52.1 \%$ & $91.3 \%$ & $41.9 \%$ & $<0.001$ \\
\hline & Discharge (cut-off: 76.50) & 0.765 & 0.032 & $0.703-0.827$ & $79.1 \%$ & $63.9 \%$ & $83.6 \%$ & $56.7 \%$ & $<0.001$ \\
\hline \multirow{3}{*}{9} & Lymphocyte count (\%) & & & & & & & & \\
\hline & Admission (cut-off: 15.50) & 0.267 & 0.028 & $0.213-0.322$ & $40.4 \%$ & $28.9 \%$ & $55.1 \%$ & $18.3 \%$ & $<0.001$ \\
\hline & Discharge (cut-off: 14.50) & 0.251 & 0.033 & $0.187-0.315$ & $36.0 \%$ & $29.2 \%$ & $43.3 \%$ & $23.3 \%$ & $<0.001$ \\
\hline \multirow{3}{*}{10} & Urea (mg/dl) & & & & & & & & \\
\hline & Admission (cut-off: 33.27) & 0.726 & 0.030 & $0.668-0.784$ & $76.7 \%$ & $62.3 \%$ & $87.0 \%$ & $44.9 \%$ & $<0.001$ \\
\hline & Discharge (cut-off: 60.92) & 0.860 & 0.028 & $0.805-0.915$ & $83.6 \%$ & $79.7 \%$ & $88.7 \%$ & $71.8 \%$ & $<0.001$ \\
\hline \multirow{3}{*}{11} & Creatinine (mg/dl) & & & & & & & & \\
\hline & Admission (cut-off: 1.11) & 0.719 & 0.029 & $0.662-0.777$ & $68.3 \%$ & $70.5 \%$ & $84.7 \%$ & $48.3 \%$ & $<0.001$ \\
\hline & Discharge (cut-off: 1.35) & 0.827 & 0.031 & $0.766-0.888$ & $78.1 \%$ & $82.2 \%$ & $85.8 \%$ & $73.1 \%$ & $<0.001$ \\
\hline
\end{tabular}




\section{Cureus}

12

$\begin{array}{lllllllll}\text { Admission (cut-off: 143.50) } & 0.478 & 0.037 & 0.407-0.550 & 21.2 \% & 91.8 \% & 74.1 \% & 51.2 \% & 0.522 \\ \text { Discharge (cut-off: 145.50) } & 0.791 & 0.036 & 0.720-0.862 & 60.3 \% & 93.2 \% & 79.0 \% & 84.6 \% & <0.001\end{array}$

TABLE 4: Receiver operating characteristic statistics for poor prognostic markers of COVID-19 fatalities (all-cause deaths)

COVID-19, coronavirus disease 2019; PPV, positive predictive value; NPV, negative predictive value; CRP, C-reactive protein; ICU, intensive care unit; AUC, area under the curve; $\mathrm{Cl}$, confidence interval; SE, standard error; ROC, receiver operating characteristic; LDH, lactate dehydrogenase.

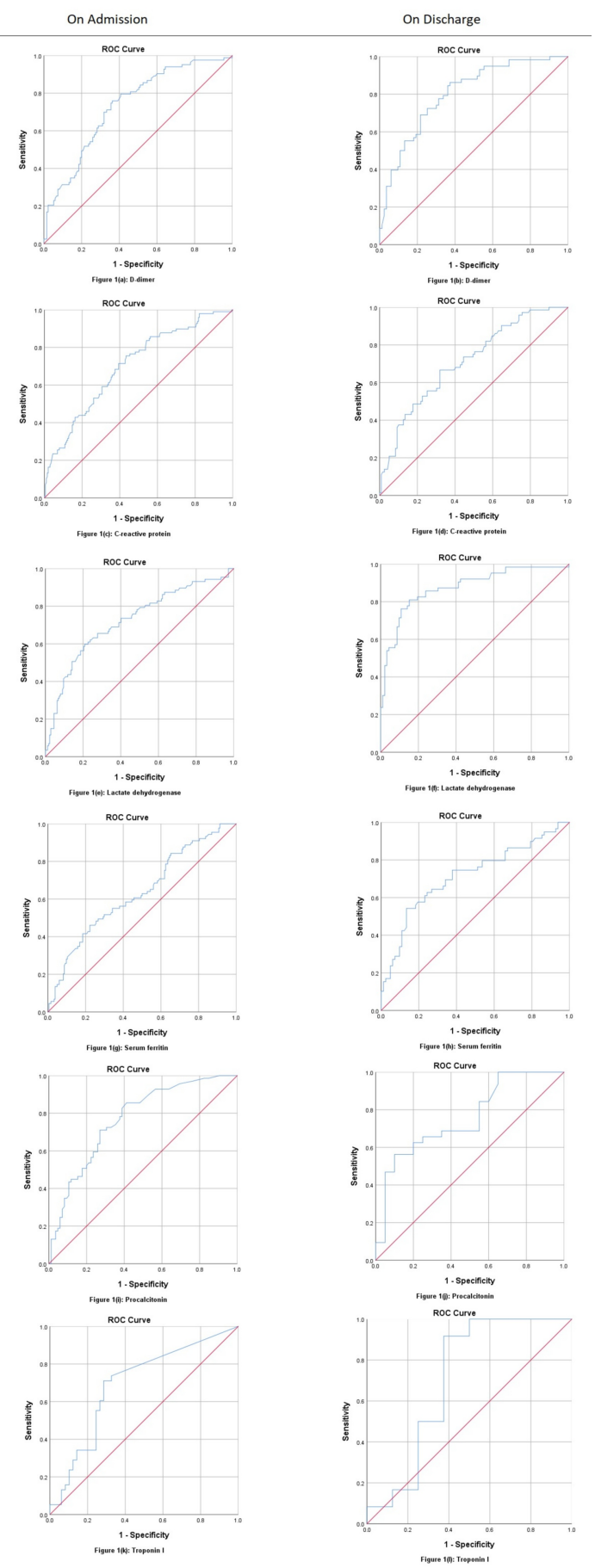

FIGURE 1: ROC curves for admitting and discharging labs of COVID-19 patients (D-dimer, CRP, LDH, ferritin, procalcitonin, and troponin I). 


\section{Cureus}

ROC, receiver operating characteristic; COVID-19, coronavirus disease 2019; CRP, C-reactive protein; LDH, lactate dehydrogenase.

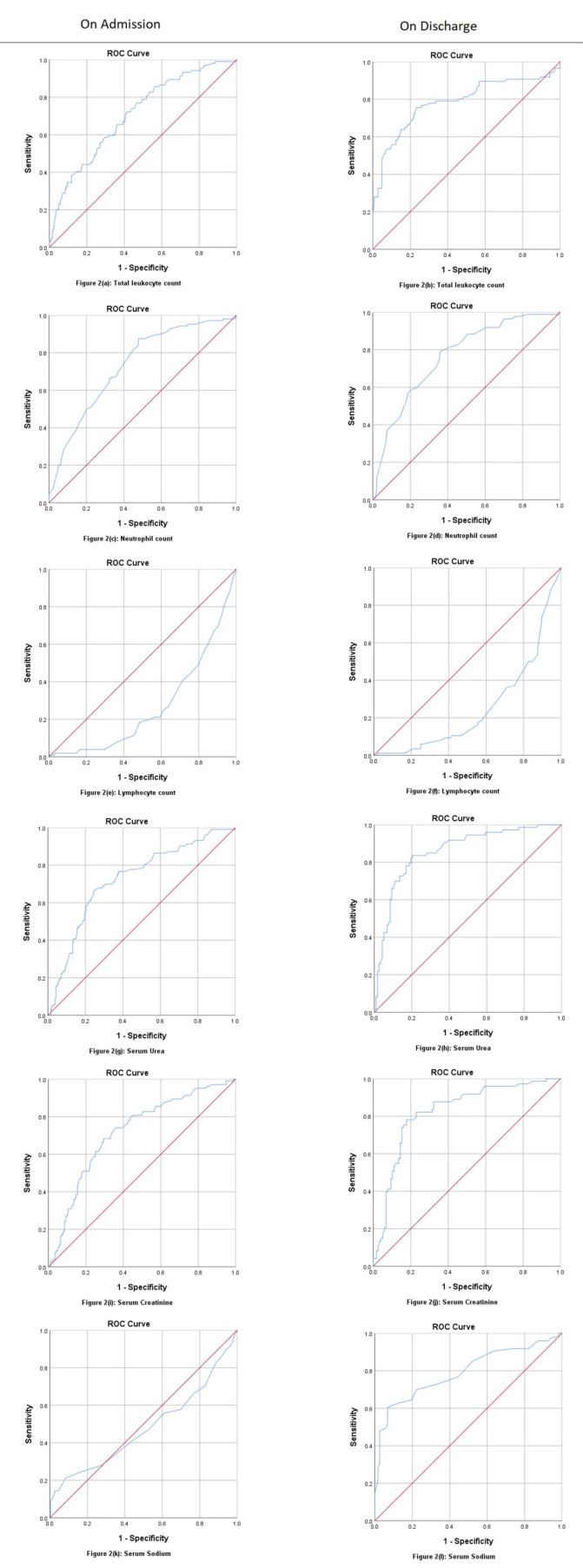

FIGURE 2: ROC curves for admitting and discharging labs of COVID-19 patients (TLC, neutrophils, lymphocytes, urea, creatinine, and sodium).

ROC, receiver operating characteristic; COVID-19, coronavirus disease 2019; TLC, total leukocyte count.

\section{Discussion}

The ongoing COVID-19 contagion has spurred researchers to explore effective disease severity predictors that can aid in combating the SARS-CoV-2 virus. The main purpose of this research was to establish biomarkers that could accurately predict the severity of coronavirus disease, thereby guiding clinicians in 
the risk stratification and clinical management of the patients. Our analysis showed that CRP is significantly elevated as compared to the mild course of the disease in severe cases of COVID-19 and was an important predictor of severity of the disease. This result is consistent with the findings of a review article that concluded that CRP amounted to $60.7 \%$ of patients infected with SARS-CoV-2 and was a crucial marker for predicting COVID-19 prognosis and mortality in these patients [19]. Qin et al. also found a significant association in their study of increased CRP and severe prognosis of the disease, but the values reported in the severe group of patients are about 2.5 times higher than ours; however, a ratio of 1.74 between the severe and non-severe groups was almost identical to our study, whereas the marker levels in the mild course of the disease are nearly identical to our levels in severe disease [20]. In their research on the use of CRP to predict disease prognosis, Gao et al. have reached the same conclusion, with CRP values reported being much higher than our analysis, although a ratio of 1:2 in the non-severe versus severe group was observed among the CRP levels close to our ratio of 1.75 [21]. A study undertaken in Wuhan, China, that evaluated the clinical characteristics of COVID-19 patients also found an important association of CRP with severe disease prognosis. However, the levels of CRP were much higher as compared to ours with a ratio of 1.67 between the severe and non-severe groups similar to our study [22]. Zheng et al. found that a mean value of $49.6 \mathrm{mg} / \mathrm{L} \mathrm{for}$ CRP was significant for a severe prognosis of the disease, which was higher than our mean of $19.86 \mathrm{mg} / \mathrm{L}$ [11]. The conclusion reached by Zheng et al. identifying CRP as a significant marker for the severe manifestation of COVID-19 is similar to ours; however, the ratio reported between severe and non-severe cases in their study is much higher compared to our study. Their values of milder disease, along with another study, were similar to our findings [11,23].

Our study showed that LDH is also significantly increased in patients experiencing a severe course of the disease compared to those with mild infections, thereby demonstrating its role as the most potential biomarker in predicting COVID-19 severity. A study conducted in Changsha also observed LDH as an important biomarker for disease severity [23]. In that study, LDH were significantly elevated in patients with severe COVID-19; however, their mean values were three times lower as compared to our reported findings. Chen et al. also documented an association of elevated levels of LDH in patients with disease severity [24]. Their study's levels of LDH were two times lower in both the severe and moderate groups of patients as compared to ours, but their research, as well as ours, concluded a substantial association between LDH levels and disease severity.

The significance of ferritin as a biomarker to monitor and predict disease severity as compared to CRP and LDH was much lower in our study. This conclusion differs from what is reported by many other studies, most of them identifying serum ferritin to be a significant marker for the prediction of disease severity $[24,25]$. A study in Wuhan, China, found a strong association of serum ferritin as a marker for the severe disease [20]. The levels of serum ferritin in our study are three times higher from the study conducted in Wuhan, in both surviving (mild-to-moderate) and non-surviving (severe) groups of patients. The levels of ferritin were much lower in that study compared to ours, but the association between the levels of ferritin and disease severity was closer to our study in terms of statistics, with our study reporting a p-value of 0.066 at admission compared to their p-value of 0.049 [24].

Our results also demonstrated the use of PCT to be a significant biomarker of the disease, which is dissimilar to the conclusions reached by Gao et al., where the authors did not find a significant association between PCT and disease severity [21]. On the contrary, several studies have reported a significant association in the elevated levels of PCT and disease severity $[23,26]$. This association seen in our study between PCT and its predictability of disease severity may be due to the higher rates of co-infection by bacteria and a high incidence of ventilator-associated pneumonia.

Studies have shown the role of D-dimer as an effective predictor for mortality of COVID-19 and thereby the severe course of the disease [12,27]. The values of D-dimer found in our study are significantly higher in both severe and non-severe patients when compared with the findings of other authors [24,28]. Most of the studies conducted to explore the clinical features and the role of biomarkers in predicting the severity of COVID-19 have found D-dimer to be an effective predictor, while some have associated it with increased mortality and ARDS $[24,25]$. Our study concluded D-dimer to be the second most effective biomarker after LDH in predicting the mortality alongside PCT, while CRP and ferritin were lagging behind the abovementioned biomarkers in predicting mortality. Cardiac markers (trop I and pro-B-type natriuretic peptide) were not effective in predicting the severity or mortality in our study. Also, serum fibrinogen had no role in predicting severity in our findings dissimilar to another study [21]. ROC curve analysis of the same study showed fibrinogen (AUC: 0.695), which was much higher than our study, while CRP (AUC: 0.600) was much lower than our study [21]. ROC curve for D-dimer had similar sensitivity (86\%) to predict severity at discharge in both the studies while our AUC was slightly lower than their study [21].

Regarding the baseline laboratory investigations, increased TLC, neutrophil count, urea, creatinine, sodium, and decreased lymphocyte count were all associated with disease severity and mortalities in our study, a finding similar to many previously conducted research studies in the region [29]. However, there were few limitations in our study, the major one being the confounding factor of various comorbidities that may be the cause of severe immune dysregulation in a certain group of patients. Other than that, secondary bacterial infections and MOF can also aggravate the immune dysregulation, which cannot be solely attributed to the viral agent being studied. 


\section{Conclusions}

We studied the effect of various biochemical markers in the prognosis of COVID-19 and the order of effectiveness among the markers. Our findings concluded that D-dimer, PCT, and LDH were superior to serum ferritin and CRP as an effective biomarker in predicting the fatality of COVID-19. We also could not establish significant associations of various other biomarkers in predicting the severity of coronavirus disease. Acute kidney injury and hypernatremia were also proven fatal events during the hospital course in our study. Such findings are crucial and can be used as guidelines when assessing the severity of the disease or treating patients in this region with the disease.

\section{Additional Information}

\section{Disclosures}

Human subjects: All authors have confirmed that this study did not involve human participants or tissue. Animal subjects: All authors have confirmed that this study did not involve animal subjects or tissue. Conflicts of interest: In compliance with the ICMJE uniform disclosure form, all authors declare the following: Payment/services info: All authors have declared that no financial support was received from any organization for the submitted work. Financial relationships: All authors have declared that they have no financial relationships at present or within the previous three years with any organizations that might have an interest in the submitted work. Other relationships: All authors have declared that there are no other relationships or activities that could appear to have influenced the submitted work.

\section{References}

1. Jin Y, Yang H, Ji W, Wu W, Chen S, Zhang W, Duan G: Virology, epidemiology, pathogenesis, and control of COVID-19. Viruses. 2020, 12:372. 10.3390/v12040372

2. Shereen MA, Khan S, Kazmi A, Bashir N, Siddique R: COVID-19 infection: origin, transmission, and characteristics of human coronaviruses. J Adv Res. 2020, 24:91-98. 10.1016/j.jare.2020.03.005

3. Vetter P, Vu DL, L'Huillier AG, Schibler M, Kaiser L, Jacquerioz F: Clinical features of COVID-19. BMJ. 2020, 369:m1470. 10.1136/bmj.m1470

4. Kernan KF, Carcillo JA: Hyperferritinemia and inflammation. Int Immunol. 2017, 29:401-409. 10.1093/intimm/dxx031

5. Giemza-Stokłosa J, Islam MA, Kotyla PJ: Hyperferritinaemia: an iron sword of autoimmunity. Curr Pharm Des. 2019, 25:2909-2918. 10.2174/1381612825666190709202804

6. Fan SL, Miller NS, Lee J, Remick DG: Diagnosing sepsis: the role of laboratory medicine. Clin Chim Acta. 2016, 460:203-210. 10.1016/j.cca.2016.07.002

7. Gabay C, Kushner I: Acute-phase proteins and other systemic responses to inflammation. N Engl J Med. 1999, 340:448-454. 10.1056/NEJM199902113400607

8. Yuki K, Fujiogi M, Koutsogiannaki S: COVID-19 pathophysiology: a review . Clin Immunol. 2020, 215:108427. 10.1016/j.clim.2020.108427

9. Teijaro JR: Cytokine storms in infectious diseases. Semin Immunopathol. 2017, 39:501-503. 10.1007/s00281-017-0640-2

10. Tan C, Huang Y, Shi F, et al.: C-reactive protein correlates with computed tomographic findings and predicts severe COVID-19 early. J Med Virol. 2020, 92:856-862. 10.1002/jmv.25871

11. Zheng Y, Xu H, Yang M, et al.: Epidemiological characteristics and clinical features of 32 critical and 67 noncritical cases of COVID-19 in Chengdu. J Clin Virol. 2020, 127:104366. 10.1016/j.jcv.2020.104366

12. Chen T, Wu D, Chen H, et al.: Clinical characteristics of 113 deceased patients with coronavirus disease 2019: retrospective study. BMJ. 2020, 368:m1091. 10.1136/bmj.m1091

13. Falcão Gonçalves P, Menezes Falcão L, Duque Pinheiro I: Procalcitonin as biomarker of infection: implications for evaluation and treatment. Am J Ther. 2017, 24:243-249. 10.1097/MJT.0000000000000210

14. Grondman I, Pirvu A, Riza A, Ioana M, Netea MG: Biomarkers of inflammation and the etiology of sepsis . Biochem Soc Trans. 2020, 48:1-14. 10.1042/BST20190029

15. Lippi G, Plebani M: Procalcitonin in patients with severe coronavirus disease 2019 (COVID- 19): a metaanalysis. Clin Chim Acta. 2020, 505:190-191. 10.1016/j.cca.2020.03.004

16. Zein JG, Lee GL, Tawk M, Dabaja M, Kinasewitz GT: Prognostic significance of elevated serum lactate dehydrogenase (LDH) in patients with severe sepsis. Chest. 2004, 126:873. 10.1378/chest.126.4_MeetingAbstracts.873S

17. Rodelo JR, De la Rosa G, Valencia ML, et al.: D-dimer is a significant prognostic factor in patients with suspected infection and sepsis. Am J Emerg Med. 2012, 30:1991-1999. 10.1016/j.ajem.2012.04.033

18. Bhutta ZA, Basnyat B, Saha S, Laxminarayan R: COVID-19 risks and response in South Asia . BMJ. 2020, 368:m1190. 10.1136/bmj.m1190

19. Siordia JA Jr: Epidemiology and clinical features of COVID- 19: a review of current literature . J Clin Virol. 2020, 127:104357. 10.1016/j.jcv.2020.104357

20. Qin C, Zhou L, Hu Z, et al.: Dysregulation of immune response in patients with COVID-19 in Wuhan, China . Clin Infect Dis. 2020, 71:762-768. 10.1093/cid/ciaa248

21. Gao Y, Li T, Han M, et al.: Diagnostic utility of clinical laboratory data determinations for patients with the severe COVID-19. J Med Virol. 2020, 92:791-796. 10.1002/jmv.25770

22. Zhang IJ, Dong X, Cao YY, et al.: Clinical characteristics of 140 patients infected with SARS-CoV-2 in Wuhan, China. Allergy. 2020, 75:1730-1741. 10.1111/all.14238

23. Zheng F, Tang W, Li H, Huang YX, Xie YL, Zhou ZG: Clinical characteristics of 161 cases of coronavirus disease 2019 (COVID-19) in Changsha. Eur Rev Med Pharmacol Sci. 2020, 24:3404-3410. 10.26355/eurrev_202003_20711 


\section{Cureus}

24. Chen G, Wu D, Guo W, et al.: Clinical and immunological features of severe and moderate coronavirus disease 2019. J Clin Invest. 2020, 130:2620-2629. 10.1172/JCI137244

25. Velavan TP, Meyer CG: Mild versus severe COVID- 19: laboratory markers. Int J Infec Dis. 2020, 95:304-307. 10.1016/j.ijid.2020.04.061

26. Liu F, Li L, Xu M, et al.: Prognostic value of interleukin-6, C-reactive protein, and procalcitonin in patients with COVID-19. J Clin Virol. 2020, 127:104370. 10.1016/j.jcv.2020.104370

27. Zhou F, Yu T, Du R, et al.: Clinical course and risk factors for mortality of adult inpatients with COVID-19 in Wuhan, China: a retrospective cohort study. Lancet. 2020, 395:1054-1062. 10.1016/S01406736(20)30566-3

28. Zhang G, Hu C, Luo L, et al.: Clinical features and short-term outcomes of 221 patients with COVID-19 in Wuhan, China. J Clin Virol. 2020, 127:104364. 10.1016/j.jcv.2020.104364

29. Asghar MS, Haider Kazmi SJ, Ahmed Khan N, et al.: Clinical profiles, characteristics, and outcomes of the first 100 admitted COVID-19 patients in Pakistan: a single-center retrospective study in a tertiary care hospital of Karachi. Cureus. 2020, 12:e8712. 